\title{
Improving Stator Winding Fault Diagnosis IN INDUCTION MOTOR USING DEMPSTER-SHAFER THEORY
}

\author{
Reza Gholamshahi ${ }^{1}$, Javad Poshtan ${ }^{2}$ and Majid Poshtan ${ }^{3}$ \\ ${ }^{1}$ Faculty of Electrical Engineering, Iran University of Science and Technology \\ Reza.gholamshahi68@gmail.com \\ ${ }^{2}$ Faculty of Electrical Engineering, Iran University of Science and Technology \\ jposhtaneiust.ac.ir \\ ${ }^{3}$ Faculty of Electrical Engineering, American University in Dubai \\ mposhtan@aud.edu
}

\begin{abstract}
Due to the important role of induction motors in industry, reliability in fault detection of these motors is of great importance. In this paper, a method is proposed for increasing the reliability of stator winding fault diagnosis using Dempster-Shafer theory. Out of the measured current and vibration signals, some features are extracted and selected according to the method, and a neural network based on DempsterShafer theory is trained by the selected features. The proposed method is then tested for various fault. Finally it is shown how data fusion can increase the accuracy of fault diagnosis. The system performance is also evaluated on a laboratory motor showing satisfactory results.
\end{abstract}

\section{KEYWORDS}

Dempster-Shafer theory, fault diagnosis, stator winding fault, induction motor.

\section{INTRODUCTION}

Today, induction motors are key tools in production lines because they can create subsequent drastic changes in human life by easing and accelerating production processes and regular services. Although induction motors can be trusted, they are always exposed to sudden unexpected failures which can even lead to stopping the production line. With the development of various industries, reducing shutdown time is becoming more important now. Accordingly, detecting and identifying the faults occurring in the system is very vital. Fault diagnosis systems are considered the tools for maintaining and protecting the processes against faults by preparing the information required for their exact evaluation. In the classical methods of fault diagnosis, a signal is used for making decision which may make faults diagnosis impossible or even stop the operation in some cases for no reason. Thus, it is essential to increase reliability of the fault diagnosis system. One of the ways for such an increase is to use multi-sensor data fusion theory[1].

On the other hand, in condition-based maintenance, a machine will be repaired or serviced only when the intelligent system notifies the undesired function. Strict implementation of such a process requires fusing data from different sensors, extracting features, classifying and using prediction algorithms. Estimating the operation condition and forecasting the remaining useful life of the machine need calculation of more than one feature [2]. 
Data fusion includes theory, techniques and tools for extracting information from different synergy sources, sensors, databases, humans, etc. Final decision or action based on the data fusion compared to the decision extracted from information of each sensor is more appropriate. However, in some situations, conflicting information and sensors with different degrees of trust might be encountered. However, the data on fusion lack accuracy and are not standardized. To solve this problem, several models have been proposed, among which probability theory, evidence theory based on the belief function and possibility theory can be pointed out [3].

Great efforts have also been made on fault diagnosis of rotating machinery using data fusion; for example, the diagnosis of bearing faults using the information fusion of fuzzy measurement and fuzzy integral at two different levels of feature fusion and taking decision fusion can be pointed out in this regard[4]. Also a method was presented in [5] for bearing fault diagnosis using the fusion of two primary sensors: an accelerometer and a load cell. After calculation of signal indices, principal component analysis was employed for redundancy reduction. For identifying the condition of the motor, K-Nearest Neighbour (KNN) classifier has been used [5]. A multi-component method for classifying fault signal based on sensor fusion data using support vector machine (SVM) and short term of the Fourier transform were proposed and tested on opening and closing valves of motors for air excavation[6]. For fault diagnosis of induction motor using transient stator current signal, [7] has proposed a decision-level fusion model by combining the information of multi-level wavelet decomposition. Also a data fusionbased misalignment fault identification algorithm is presented, which isolates fault features from similar features generated because of other operating conditions for a 5.5 horse-power induction motor in [8].

The information gathered from various sensors can be used and fused by Dempster-Shafer theory in order to investigate other faults such as faults in the motor piston [9] and mechanical faults in induction motors $[3,10]$. Also, a novel induction motor fault diagnosis system and condition monitoring using Dempster-Shafer based on industrial wireless sensor networks were presented respectively in [11] and [12].

This paper presents a stator winding fault diagnosis method for induction motors based on Dempster-Shafer theory by using stator current and vibration signals. At first, the features of measured signal are extracted using signal processing techniques. Then a neural network scheme with Dempster- Shafer theory based on Denoeux's works [13] is used for classifying the extracted features. Finally, estimated quantities from vibration and current classifiers are fused by Dempster-Shafer theory for increasing the diagnostic accuracy.

This paper is organized as follows. After a brief description of Dempster- Shafer theory in section 2, we explain the neural network based on Dempster-Shafer theory in section 3. The feature extraction method is described in section 4. The proposed fault diagnosis method is presented in section 5 and the results are presented in section 6 .

\section{DEMPSTER-SHAFER THEORY}

Initially, Evidence Theory was founded on lower and upper probability distribution by Dempster. According to these mathematical bases, Shafer demonstrated the ability of the belief functions for modelling uncertain information. Basic concepts and mechanisms of DempsterShafer theory is introduced below [3].

Assume $\Omega=\left\{A_{1}, A_{2}, \ldots, A_{N}\right\}$ is a finite non-empty set of mutually $\mathrm{N}$ exhaustive and exclusive

hypotheses related to a problem domain called frame of discernment. Then $2^{\Omega}$ denotes the power set composed of $2^{N}$ proposition $\mathrm{A}$ of $\Omega$, that is: 


$$
2^{\Omega}=\left\{\phi,\left\{A_{1}\right\},\left\{A_{2}\right\}, \ldots,\left\{A_{N}\right\},\left\{A_{1} \cup A_{2}\right\},\left\{A_{1} \cup A_{3}\right\}, \ldots, \Omega\right\}
$$

Basic belief assignment (BBA) is the key point of Dempster-Shafer evidence theory. Mass of belief in an element of $\Omega$ is very similar to probability distribution; however, unit mass is distributed among elements of $2^{\Omega}$. The mass of belief $m_{j}$ assigned to the information source $S_{j}$ is defined by $m_{j}: 2^{\Omega} \rightarrow[0,1]$, which verifies the following properties:

$m_{j}(\phi)=0 ; \sum_{A \subseteq \Omega} m_{j}(A)=1$

The mass $m_{j}(A)$ indicates the strength with which the evidence $S_{j}$ supports A. Each subset $A \subseteq \Omega$ such as $m_{j}(A)>0$ is called focal element of $\mathrm{m}$. The belief function $\operatorname{Bel}_{j}(A)$ and plausibility function $P l_{j}(A)$ are defined for all $A \subseteq \Omega$ respectively as

$$
\begin{aligned}
& \operatorname{Bel}_{j}(A)=\sum_{B \subseteq A} m_{j}(B) \\
& P l_{j}(A)=1-\operatorname{Bel}_{j}(\bar{A})=\sum_{A \cap B=\phi} m_{j}(B)
\end{aligned}
$$

where $\bar{A}$ is negation of hypothesis A. In fact, $\operatorname{Bel}_{j}(A)$ shows necessary (minimal) support for $\mathrm{A}$ and could be interpreted as a global measure of a belief that hypothesis $\mathrm{A}$ is true. Belief of $\mathrm{A}$ can be defined as sum of all the belief masses allocated to hypothesis B. But, $P l_{j}(A)$ gives potential (maximal) support for A and is sum of the parts of belief which are allocated to hypothesis B and are compatible with A.

According to the basic belief assignment $\left(m_{j}\right)$ for each information source $S_{j}$, using a combination rule is possible for providing combined masses which synthesize knowledge of different sources. These belief masses can be then applied by a decision process which has the benefit of containing the whole knowledge in the belief functions given by each source. Evidence can be combined by calculating orthogonal sum using Dempster's rule of combination, in which evidence A and evidence B are used for calculating a new belief function for a focal element $\mathrm{C}$ as

$m(C)=m_{1} \oplus m_{2}(C)=(1-K)^{-1} \sum_{A \cap B=C} m_{1}(A) m_{2}(B)$

where

$$
K=\sum_{A \cap B=\phi} m_{1}(A) m_{2}(B)
$$

According to the combination rule, demonstrating the combination of $m_{1}$ and $m_{2}$ allocates total amount of belief among the subsets $\Omega$ by assigning $m_{1}(A) m_{2}(B)$ to the set resulting from the interaction of sets $\mathrm{A}$ and $\mathrm{B}$. $\mathrm{K}$ which is a normalization factor is often interpreted as a measure of conflict between the two sources. With increasing $\mathrm{K}$, the sources have more conflict and their combinations become less relevant.

A belief function has to be transformed into a probability function for decision-making. The only transformation which satisfies elementary rationality requirements is the pignistic 
transformation given by Smets and Kennes [14], in which each mass of belief $m(A)$ is equally distributed among elements of A for all $A \subseteq \Omega$, which leads to pignistic probability distribution of class $\omega$ defined as

$\operatorname{BetP}(\omega)=\sum_{\omega \in A} \frac{m(A)}{|A|}, \forall \omega \in \Omega$

where $|A|$ denotes cardinality of $A \subseteq \Omega$.

\section{NeUral network Classifier}

In this paper, a neural network based on Dempster-Shafer theory is used as a classifier which includes one input layer $\left(L_{1}\right)$, two hidden layers $\left(L_{2}\right.$ and $\left.L_{3}\right)$ and one output layer $\left(L_{4}\right)$, as given in Figure 1.

The classification procedure which uses the trained NN was recently proposed in [13] as follows:

Step 1: Calculating the Euclidean distances $d^{i}$ between the input vector $\boldsymbol{x}$ and each prototype vector $\boldsymbol{p}^{i}$ :

$d^{i}=\left\|\boldsymbol{x}-\boldsymbol{p}^{i}\right\|, \quad i=1, \ldots, n$.

The information provided by each prototype is given by a BBA $m^{i}$, which depends on the class membership of $\boldsymbol{p}^{i}$ and also on $d^{i}$.

Step 2: Calculating the output of the first hidden layer by activation function. Hidden layer $L_{2}$ contains $\mathrm{n}$ units with activation

$s^{i}=\alpha^{i} \exp \left(-\gamma^{i}\left(d^{i}\right)^{2}\right)$ for $i=1, \ldots, n$

Prototypes $\boldsymbol{p}^{i}, \alpha^{i}$ and $\gamma^{i}$ are previously organized by the training set and $s^{i}$ is calculated when the testing data are input. 


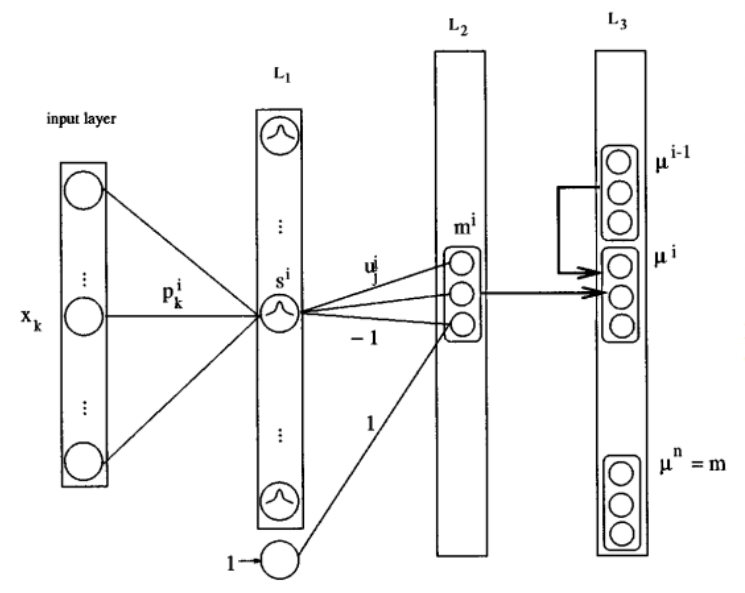

Figure 1: Neural network classifier

Step 3: Calculating the BBA's $m^{i}$ associated with each prototype in the second hidden layer $L_{3}$. It is composed of $\mathrm{n}$ modules, each with $\mathrm{M}+1$ units. Units of module $i$ are connected to neuron $i$ of the previous layer. The activation vector of module $i$ corresponds to the belief masses assigned by $m^{i}$.

$$
\begin{aligned}
m^{i} & =\left(m^{i}\left(\left\{\omega_{1}\right\}\right), \ldots, m^{i}\left(\left\{\omega_{M}\right\}\right), m^{i}(\Omega)\right) \\
& =\left(u_{1}^{i} s^{i}, u_{2}^{i} s^{i}, \ldots, u_{M}^{i} s^{i}, 1-s^{i}\right)
\end{aligned}
$$

The $\mathrm{n}$ belief assignment's $m^{i}$ is then combined in the output layer $L_{4}$, composed of $\mathrm{n}$ interconnected modules with $\mathrm{M}+1$ units. Activation vector $\boldsymbol{\mu}^{i}$ of module $i$ in that layer is defined as:

$$
\boldsymbol{\mu}^{i}=\left(\mu^{i}\left(\left\{\omega_{1}\right\}\right), \ldots, \mu^{i}\left(\left\{\omega_{1}\right\}\right), \mu^{i}(\Omega)\right)^{T}
$$

where $\mu^{i}$ is the conjunctive combination of the BBA's $m^{1}, \ldots, m^{i}$ :

$$
\begin{aligned}
& \mu^{1}=m^{1} \\
& \mu^{i}=\bigcap_{k=1}^{i} m^{k}=\mu^{i-1} \bigcap m^{i} \quad i=2, \ldots, n
\end{aligned}
$$

The activation vectors $\mu^{i}$ for $i=2, \ldots, n$ can be recursively calculated using the following formula:

$$
\begin{aligned}
& \mu_{i}^{j}=\mu_{j}^{i-1} m_{j}^{i}+\mu_{j}^{i-1} m_{M+1}^{i}+\mu_{M+1}^{i-1} m_{j}^{i} \quad j=1, \ldots, M \\
& \mu_{M+1}^{i}=\mu_{M+1}^{i-1} m_{M+1}^{i}
\end{aligned}
$$


which can be done by each computation element in module $i>1$ of layer $L_{4}$ provided that it receives the input from module $i-1$ in the same layer and from module $i$ in layer $L_{3}$. This aim can be fulfilled by fixed-weight connections between layers $L_{3}$ and $L_{4}$.

Step 4: Decision-making on the input data class. The network output vector $\boldsymbol{m}=\left(m_{1}, \ldots, m_{M+1}\right)$ is then defined as:

$\boldsymbol{m}=\boldsymbol{\mu}^{n}$

This vector is the fusion of all BBAs.

\section{Feature Extraction}

Feature extraction of a signal is the initial step in any monitoring and fault diagnosis system and its accuracy has a direct effect on the final monitoring results. Therefore, feature extraction should maintain critical information for decision-making. But, applying too many feature parameters can be a burden to networks since calculating the results can be very timeconsuming. A feature extraction technique [15] is applied here to extract some parameters which can properly represent the fault features taken from total parameters.

Step 1: Calculating average distance between the same condition data $\left(d_{i, j}\right)$ and obtaining average distance between seven conditions $\left(d_{a i}\right)$. The equations are defined as shown below:

$$
d_{i, j}=\frac{1}{N(N-1)} \sum_{m, n=1}^{N}\left|p_{i, j}(m)-p_{i, j}(n)\right| ; \quad(m, n=1,2, \ldots, N, m \neq n)
$$

where $N$ is number of the same conditions $(N=20), p_{i, j}$ is feature value, $d_{i, j}$ is average distance of the same condition and $i$ and $j$ represent the number of parameters and conditions, respectively. Also,

$$
d_{a i}=\frac{1}{M} \sum_{j=1}^{M} d_{i, j}
$$

where $M$ is the number of different conditions $(M=7)$.

Step 2: Calculating average distance between different condition data $\left(d_{a i}^{\prime}\right)$.

$$
d_{a i}^{\prime}=\frac{1}{M(M-1)} \sum_{m, n=1}^{N}\left|p_{a i, m}-p_{a i, n}\right| ; \quad(m, n=1,2, \ldots, N, m \neq n)
$$

where $d_{a i}^{\prime}$ is average distance between different conditions data, and $p_{a i, j}$ is average value of the same condition for 20 data:

$$
p_{a i, j}=\frac{1}{N} \sum_{n=1}^{N} p_{i, j}(n)
$$


Step 3: Calculating the ratio $\alpha_{i}=d_{a i}^{\prime} / d_{a i}$.

Step 4: Selecting three feature parameters $\alpha_{i}$ from large to small values. Larger values of $\alpha_{i}$ show the feature is more properly.

\section{EXPERIMENTAL TeST}

To diagnose classify fault of a three-phase induction motor, it's current and vibration signals are employed. In this regard, performing four processes of data collection, computation and feature extraction, and training and classification of data is required. The proposed fault diagnosis system for induction motors is shown in Figure 2.

In order to test and investigate the performance of the proposed FD scheme for identifying the faults in the stator winding, a 5-horse-power three-phase induction motor stator is re-wired as shown in Fig. 2. When re-wiring, a number of the stator windings inside the stator are shortcircuited out of the motor frame. Status of the coils and the fault scenarios are listed in Table 1. After data processing, current and vibration data are sent to feature extraction unit. In this paper, statistical information of time-based data is applied for obtaining feature information from the measured signals. Several features such as mean, standard deviation, skewness, kurtosis, root mean square, crest factor, shape factor and entropy are selected. Then based on feature selection method, described in section 4, proper features are selected.

Table 1: Description of the induction motor faults

\begin{tabular}{|c|l|}
\hline symbol & \multicolumn{1}{|c|}{ Description } \\
\hline$F_{1}$ & Free fault (healthy) \\
\hline$F_{2}$ & $10 \%$ of winding stator is short circuit. \\
\hline$F_{3}$ & $7 \%$ of winding stator is short circuit. \\
\hline$F_{4}$ & $15 \%$ of winding stator is short circuit. \\
\hline$F_{5}$ & $5 \%$ of winding stator is short circuit. \\
\hline$F_{6}$ & $2 \%$ of winding stator is short circuit. \\
\hline$F_{7}$ & $20 \%$ of winding stator is short circuit. \\
\hline
\end{tabular}

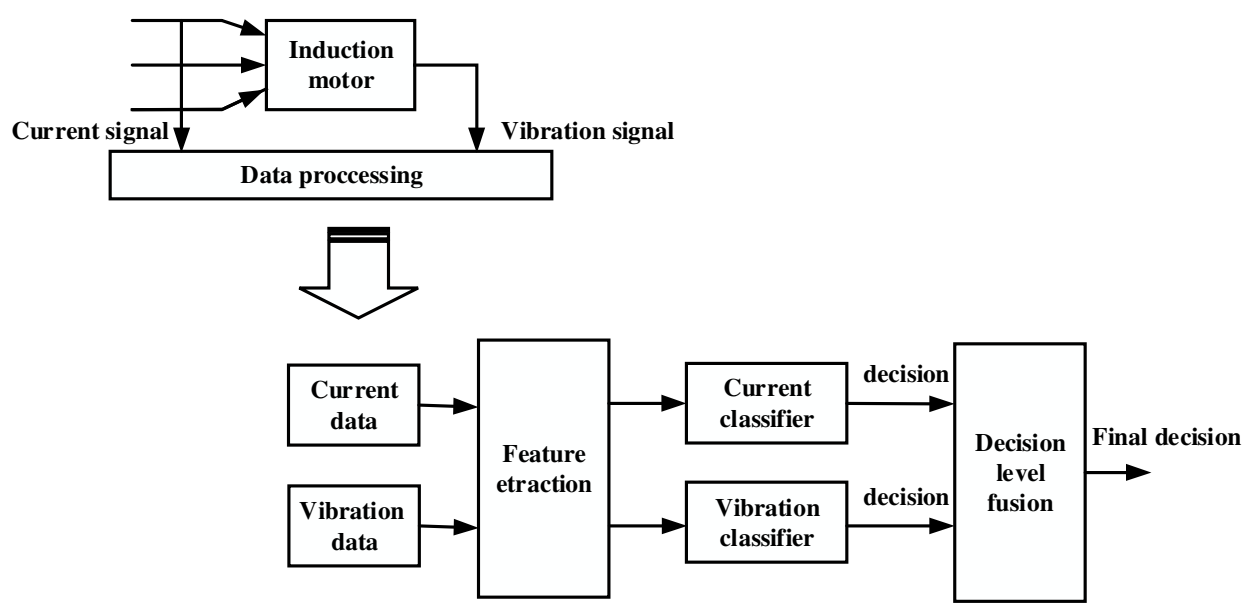

Figure 2: Flowchart of the proposed fault diagnosis system 


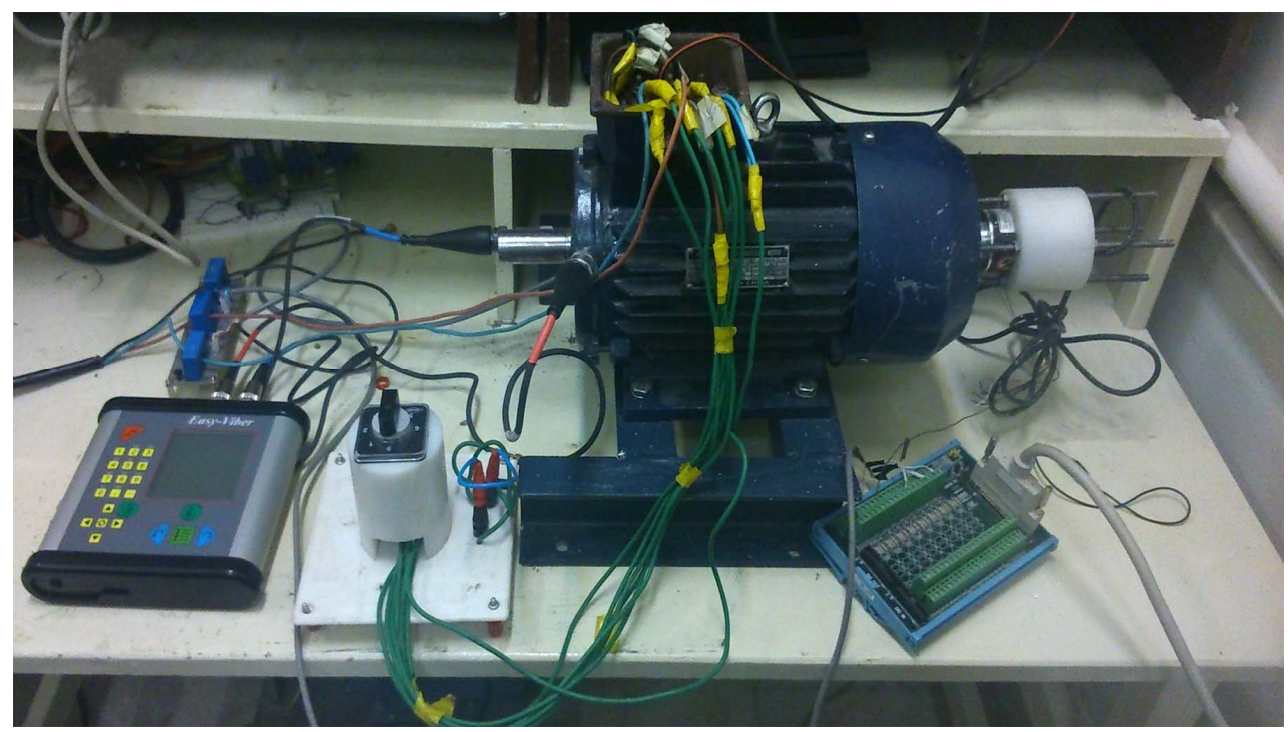

Figure 3: Laboratory setup

After extracting three proper features from the feature vectors, measured signals in each condition are used for training the neural network based on Dempster-Shafer classifier. The initial prototype locations in this classifier were specified using standard clustering procedure (k-means algorithm)[16]. After obtaining input distance from each of the prototypes, activation function value $s^{i}=\alpha^{i} \exp \left(-\gamma^{i}\left(d^{i}\right)^{2}\right)$ was computed and then BBA $\boldsymbol{m}^{i}=\left(u_{1}^{i} s^{i}, \ldots, u_{M}^{i} s^{i}, 1-s^{i}\right)$ was achieved. For initial values of matrix $\boldsymbol{u}$, membership degree of the obtained class is obtained during construction of the prototype. After calculating the BBAs, they are combined, in order to create network output. The prototypes and parameters of the classifier were optimized by a training procedure[13].In the condition classification, the set of possible hypotheses corresponded to the set of expected faulty conditions, in which $\mathrm{F}_{2}-\mathrm{F}_{7}$ denotes a particular faulty condition and $\mathrm{F}_{1}$ stands for the fault-free (healthy) condition, as demonstrated in Table 1.

\section{RESULTS}

LA-55p current sensor and data card ADVANTECH PCI-1711 were used for sampling the current. The processes for any conditions are repeated 20 times; in Figure 4-a, phase a current signal can be seen for a limited time. EASY VIBER is used for vibration data collecting and repeated for any conditions 15 times. In Figure 4-b, some of vibration signals showed. 
Electrical and Electronics Engineering: An International Journal (ELELIJ) Vol 3, No 2, May 2014

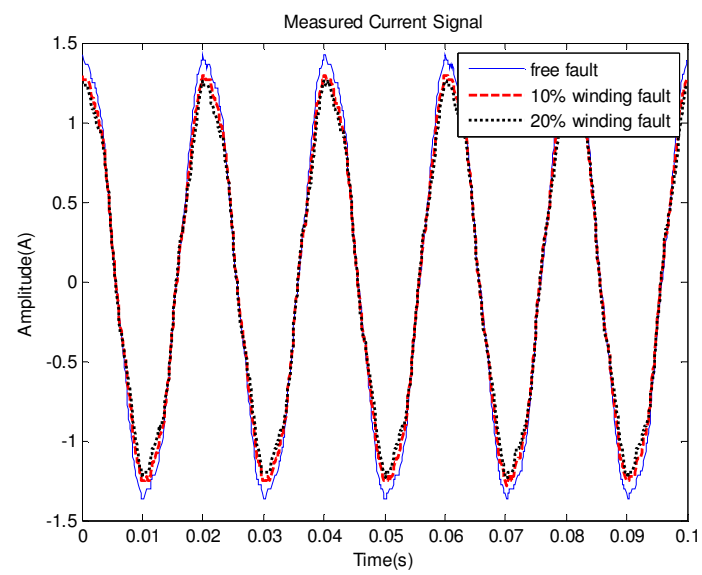

(a)
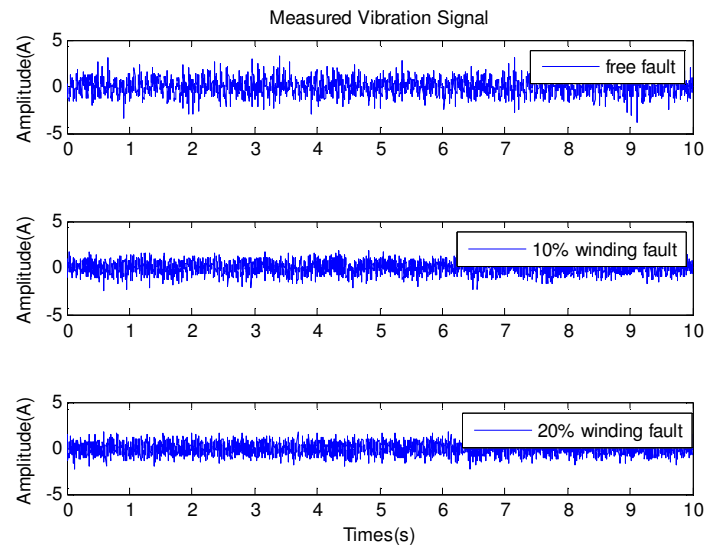

(b)

Figure 4: The measured signals in different conditions, a) current signal, b) vibration signal

After signal processing, measured signal sent to feature extraction unit. In figure 5, a number of calculated features for current and vibration signal has been shown.

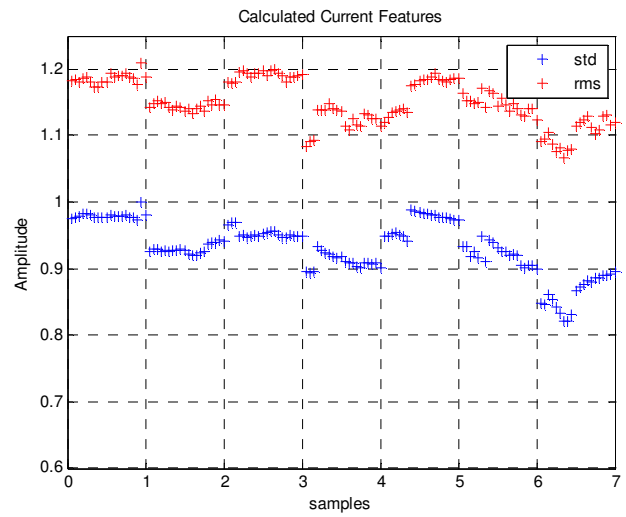

(a)

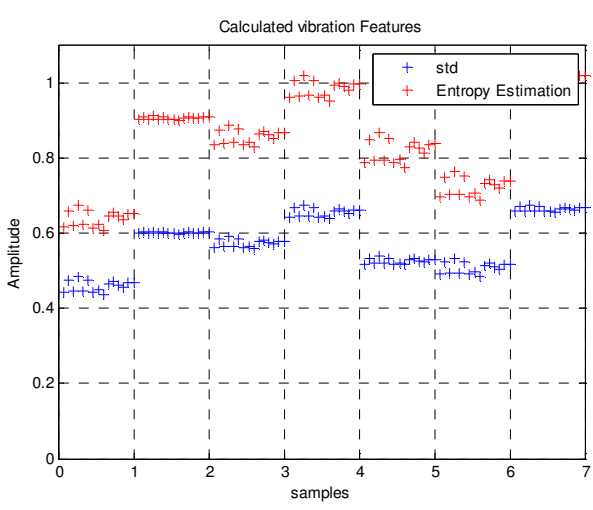

(b)

Figure 5: The calculated features for, a) current signal, b) vibration signal 
For selecting proper features, the calculated coefficient can be seen in Figure 6.

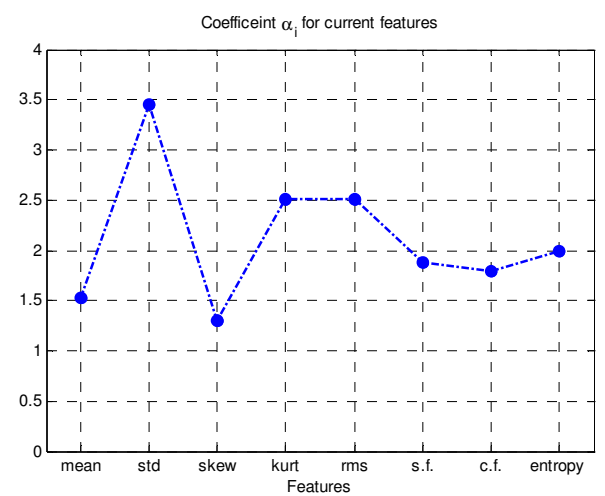

(a)

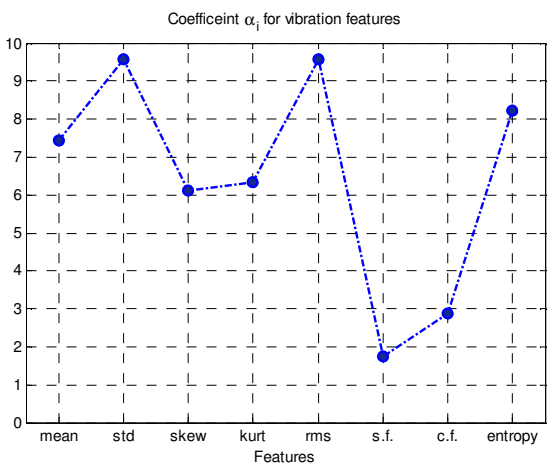

(b)

Figure 6: The coefficient $\alpha$ for, a) current features, b) vibration features

After extracting three proper features from the feature vectors calculated measured signals measured, features of 10 samples measured for training the neural network based on DempsterShafer classifier were used. Thus, a $70 \times 3$ matrix for 7 conditions and also fault class with a $70 \times 1$ vector were given to the neural network for training. After calculating the BBAs, they were combined to each other in order to create network output. By comparing the network output and the desired output, error was formed and, using descent gradient algorithm, the network parameters were trained as batch mode[13]. After network training, features of remaining samples were employed in each condition for testing. All the data were given as input to the trained neural network and the final result is obtained and presented.

Table 2 show classification results for the current classifier system. For example, for the experimental data $F_{4}$ the neural network output for the first phase current was $F_{2}>F_{4}>F_{5}>F_{1}>F_{3}>F_{7}>F O D>F_{6}$, which indicates failure in correct detection of fault. FOD (frame of discernment) represents lack of detection of the input data class.

Table 2: BBA's output current classifier

\begin{tabular}{|l|l|l|l|l|l|l|l|l|}
\hline \multirow{2}{*}{$\begin{array}{l}\text { Test } \\
\text { pattem }\end{array}$} & \multicolumn{6}{|l|}{ Classification results } \\
\cline { 2 - 9 } & $F_{1}$ & $F_{2}$ & $F_{3}$ & $F_{4}$ & $F_{5}$ & $F_{6}$ & $F_{7}$ & $F O D$ \\
\hline$F_{1}$ & 0.6333 & 0.0644 & 0.1925 & 0.0208 & 0.0511 & 0.0104 & 0.0058 & 0.0219 \\
\hline$F_{2}$ & 0.0145 & 0.8358 & 0.0069 & 0.0118 & 0.1118 & 0.0108 & 0.0027 & 0.0058 \\
\hline$F_{3}$ & 0.6232 & 0.0542 & 0.2409 & 0.0065 & 0.0426 & 0.0107 & 0.0017 & 0.0202 \\
\hline$F_{4}$ & 0.0298 & 0.5339 & 0.0215 & 0.2717 & 0.0950 & 0.0147 & 0.0177 & 0.0157 \\
\hline$F_{5}$ & 0.0227 & 0.0556 & 0.2807 & 0.0060 & 0.6219 & 0.0059 & 0.0013 & 0.0059 \\
\hline$F_{6}$ & 0.0213 & 0.0496 & 0.0087 & 0.0346 & 0.0569 & 0.7787 & 0.0155 & 0.0347 \\
\hline$F_{7}$ & 0.0030 & 0.0192 & 0.0017 & 0.2352 & 0.0129 & 0.0142 & 0.6863 & 0.0275 \\
\hline
\end{tabular}

Tables 3 show classification results for the vibration classifier system. For example, for the test data $F_{5}$ the neural network output for the vibration was $F_{5}>F_{3}>F_{6}>F O D>F_{2}>F_{1}>F_{4}>F_{7}$, which means the fault can be diagnosed correctly. 
Electrical and Electronics Engineering: An International Journal (ELELIJ) Vol 3, No 2, May 2014

Table 3: BBA's output vibration classifier

\begin{tabular}{|l|l|l|l|l|l|l|l|l|}
\hline \multirow{2}{*}{$\begin{array}{l}\text { Test } \\
\text { patter }\end{array}$} & \multicolumn{6}{|l|}{ Classification results } \\
\cline { 2 - 9 } & $F_{1}$ & $F_{2}$ & $F_{3}$ & $F_{4}$ & $F_{5}$ & $F_{6}$ & $F_{7}$ & $F O D$ \\
\hline$F_{1}$ & 0.7974 & $7.8 \mathrm{e}-5$ & 0.0008 & $7.3 \mathrm{e}-7$ & 0.0050 & 0.1061 & $5.2 \mathrm{e}-8$ & 0.0907 \\
\hline$F_{2}$ & $3.3 \mathrm{e}-7$ & 0.9928 & 0.0039 & 0.0012 & 0.0006 & $3.0 \mathrm{e}-5$ & 0.0004 & 0.0011 \\
\hline$F_{3}$ & 0.0002 & 0.0941 & 0.7449 & 0.0047 & 0.1267 & 0.0056 & 0.0013 & 0.0226 \\
\hline$F_{4}$ & $4.6 \mathrm{e}-9$ & 0.0062 & 0.0012 & 0.5444 & 0.0002 & $2.2 \mathrm{e}-6$ & 0.4310 & 0.0170 \\
\hline$F_{5}$ & 0.0060 & 0.0310 & 0.1403 & 0.0022 & 0.6141 & 0.1348 & 0.0005 & 0.0710 \\
\hline$F_{6}$ & 0.0515 & 0.0010 & 0.0059 & $2.8 \mathrm{e}-5$ & 0.0268 & 0.8755 & $3.5 \mathrm{e}-6$ & 0.0393 \\
\hline$F_{7}$ & $9.2 \mathrm{e}-10$ & 0.0034 & 0.0006 & 0.0719 & $7.2 \mathrm{e}-5$ & $7.1 \mathrm{e}-7$ & 0.9114 & 0.0126 \\
\hline
\end{tabular}

Therefore, to improve performance of classification and fault diagnosis, output combination of current and vibration classifying systems of the motor was used by Dempster-Shafer theory. Also, various architectures of data level fusion, feature level fusion and decision level fusion could be used, depending on the type of sensors. In this paper, feature level fusion was used to improve accuracy of classification and fault diagnosis. To do this, first, classifiers were individually trained for classifying input data and the outputs were combined with each other according to Dempster-Shafer rule. Table 4 lists results of combining current and vibration classifiers for the experimental patterns. Table 4 illustrates that the fusion of current and vibration classifiers increased the accuracy of fault diagnosis.

\section{Conclusions}

This paper presented a stator winding fault diagnosis method for induction motors based on Dempster-Shafer theory by using stator current and vibration signals. Similar to neural networks, classification was conducted based on similarity of input pattern to a set of reference patterns. This information was transformed into BBA functions that were ultimately combined with each other based on Dempster-Shafer theory. The results indicated that this method could increase accuracy in fault diagnosis.

Table 4: BBA's output fusion of two classifier

\begin{tabular}{|l|l|l|l|l|l|l|l|l|}
\hline \multirow{2}{*}{$\begin{array}{l}\text { Test } \\
\text { pattem }\end{array}$} & \multicolumn{6}{|l}{ Classification results } \\
\cline { 2 - 9 } & $F_{1}$ & $F_{2}$ & $F_{3}$ & $F_{4}$ & $F_{5}$ & $F_{6}$ & $F_{7}$ & $F O D$ \\
\hline$F_{1}$ & 0.9400 & 0.0095 & 0.286 & 0.0031 & 0.0081 & 0.0071 & 0.0009 & 0.0032 \\
\hline$F_{2}$ & $1.9 \mathrm{e}-5$ & 0.9997 & $6.8 \mathrm{e}-5$ & $4.2 \mathrm{e}-5$ & 0.0002 & $1.5 \mathrm{e}-5$ & $7.4 \mathrm{e}-6$ & $7.8 \mathrm{e}-6$ \\
\hline$F_{3}$ & 0.0610 & 0.0354 & 0.8600 & 0.0012 & 0.0384 & 0.0018 & 0.0003 & 0.0020 \\
\hline$F_{4}$ & 0.0027 & 0.0654 & 0.0021 & 0.8418 & 0.0086 & 0.0013 & 0.0768 & 0.0014 \\
\hline$F_{5}$ & 0.0036 & 0.0117 & 0.1202 & 0.0009 & 0.8586 & 0.0040 & 0.0002 & 0.0008 \\
\hline$F_{6}$ & 0.0049 & 0.0027 & 0.0008 & 0.0018 & 0.0062 & 0.9810 & 0.0008 & 0.0018 \\
\hline$F_{7}$ & $5.5 \mathrm{e}-5$ & 0.0006 & $5.8 \mathrm{e}-5$ & 0.0320 & 0.0002 & 0.0003 & 0.9663 & 0.0005 \\
\hline
\end{tabular}


The features extracted from three-phase current of the motor denoted its situation using classifying input data. Classifying results of faulty conditions was numerically expressed and Dempster-Shafer rule was used to obtain their combination. Results of three classifiers were complementary, which caused more reliable results in fault diagnosis.

Table 5: Comparing the classifiers

\begin{tabular}{|l|l|l|l|l|l|l|l|l|}
\hline \multirow{2}{*}{ Test pattem } & \multicolumn{6}{|l|}{ Classification results } \\
\cline { 2 - 9 } & $F_{1}$ & $F_{2}$ & $F_{3}$ & $F_{4}$ & $F_{5}$ & $F_{6}$ & $F_{7}$ & Average \\
\hline Current classifier & 0.6333 & 0.8358 & 0.2409 & 0.2717 & 0.6214 & 0.7787 & 0.6863 & 0.5812 \\
\hline Vibration classifier & 0.7974 & 0.9928 & 0.7449 & 0.5444 & 0.6141 & 0.8755 & 0.9114 & 0.7829 \\
\hline Fusion classifier & 0.9400 & 0.9997 & 0.8600 & 0.8418 & 0.8586 & 0.9810 & 0.9663 & 0.9211 \\
\hline
\end{tabular}

\section{REFERENCES}

[1] J. Faiz, B. M. Ebrahimi, B. Akin, and H. A. Toliyat, "Finite-element transient analysis of induction motors under mixed eccentricity fault," IEEE Transactions on Magnetics, vol. 44, pp. 66-74, 2008.

[2] K. M. Reichard, M. Van Dyke, and K. Maynard, "Application of sensor fusion and signal classification techniques in a distributed machinery condition monitoring system," in AeroSense, pp. 329-336, 2000.

[3] B.-S. Yang and K. J. Kim, "Application of Dempster-Shafer theory in fault diagnosis of induction motors using vibration and current signals," Mechanical Systems and Signal Processing, vol. 20, pp. 403-420, 2006.

[4] X. Liu, L. Ma, and J. Mathew, "Rotating machinery fault diagnosis based on fuzzy data fusion techniques," 2007.

[5] M. Safizadeh and S. Latifi, "Using multi-sensor data fusion for vibration fault diagnosis of rolling element bearings by accelerometer and load cell," Information Fusion, vol. 18, pp. 1-8, 2014.

[6] T. P. Banerjee and S. Das, "Multi-sensor data fusion using support vector machine for motor fault detection," Information Sciences, vol. 217, pp. 96-107, 2012.

[7] G. Niu, A. Widodo, J.-D. Son, B.-S. Yang, D.-H. Hwang, and D.-S. Kang, "Decision-level fusion based on wavelet decomposition for induction motor fault diagnosis using transient current signal," Expert Systems with Applications, vol. 35, pp. 918-928, 2008.

[8] S. B. Chaudhury, M. Sengupta, and K. Mukherjee, "Experimental study of induction motor misalignment and its online detection through data fusion," Electric Power Applications, IET, vol. 7, pp. 58-67, 2013.

[9] O. Basir and X. Yuan, "Engine fault diagnosis based on multi-sensor information fusion using Dempster-Shafer evidence theory," Information Fusion, vol. 8, pp. 379-386, 2007.

[10] B. Leping, L. K. Pei, and H. Xinguo, "The Rotor Fault Diagnosis Method of the Induction Motor Based on the DS Evidential Theory," in Asia-Pacific Power and Energy Engineering Conference (APPEEC), pp. 1-4, 2011.

[11] L. Hou and N. W. Bergmann, "Induction motor fault diagnosis using industrial wireless sensor networks and Dempster-Shafer classifier fusion," in 37th Annual Conference on IEEE Industrial Electronics Society, pp. 2992-2997, 2011.

[12] L. Hou and N. W. Bergmann, "Novel industrial wireless sensor networks for machine condition monitoring and fault diagnosis," IEE Transaction on Instrumentation and Measurement, vol. 61, pp. 2787- 2798, 2012.

[13] T. Denoeux, "A neural network classifier based on Dempster-Shafer theory," IEEE Transactions on Systems, Man and Cybernetics, Part A: Systems and Humans, vol. 30, pp. 131-150, 2000.

[14] P. Smets and R. Kennes, "The transferable belief model," Artificial intelligence, vol. 66, pp. 191234, 1994.

[15] B.-S. Yang, T. Han, and J. L. An, "ART-KOHONEN neural network for fault diagnosis of rotating machinery," Mechanical Systems and Signal Processing, vol. 18, pp. 645-657, 2004.

[16] T. Denoeux, "Analysis of evidence-theoretic decision rules for pattern classification," Pattern recognition, vol. 30, pp. 1095-1107, 1997. 
Electrical and Electronics Engineering: An International Journal (ELELIJ) Vol 3, No 2, May 2014

\section{Authors}

Reza Gholamshahi -received the B.Sc. degree in control engineering from Shiraz University, Shiraz, Iran, in 2011. He is currently pursuing his study towards the M.S. degree at Iran University of Science and Technology. His research interest focuses on Data Fusion and Fault diagnosis.

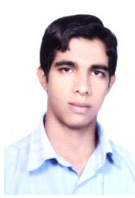

Dr. Javad Poshtan -received his B.Sc., M.Sc., and Ph.D. degrees in electrical engineering from Tehran University, Tehran, Iran, in 1987, Sharif University of Technology, Tehran, Iran, in 1991, and the University of New Brunswick, Canada, in 1997, respectively. Since 1997, he has been with the Department of Electrical Engineering at Iran University of Science and Technology. He is involved in academic and research activities in areas such as Fault diagnosis, control systems theory, system identification, and estimation theory.

Dr. Majid Poshtan - is currently an associate professor of electrical engineering in American University in Dubai, UAE. He graduated from Tulane University, USA in year 2000 and has worked in electric power system utilities and academic centers since then. Prior to joining AUD in 2011, He has taught and performed industrial research in the Petroleum Institute in Abu Dhabi for eight years. He has more than 60 publications in the area of power systems, electric machines and control systems.
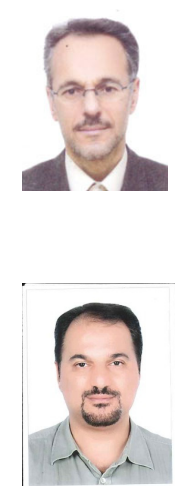